\title{
Article
}

\section{Compensation Strategies for Bioelectric Signal Changes in Chronic Selective Nerve Cuff Recordings: A Simulation Study}

\author{
Stephen Sammut ${ }^{1,2}$, Ryan G. L. Koh ${ }^{2}$ (D) and José Zariffa ${ }^{1,2,3,4, * \mathbb{D}}$ \\ 1 Institute of Biomedical Engineering, University of Toronto, Toronto, ON M5S 3G4, Canada; \\ stephen.sammut@utoronto.ca \\ 2 KITE, Toronto Rehab, University Health Network, Toronto, ON M5G 2A2, Canada; \\ ryan.koh@mail.utoronto.ca \\ 3 Edward S Rogers Sr Department of Electrical and Computer Engineering, University of Toronto, \\ Toronto, ON M4G 3V9, Canada \\ 4 Rehabilitation Sciences Institute, University of Toronto, Toronto, ON M5S 2E4, Canada \\ * Correspondence: jose.zariffa@utoronto.ca
}

Citation: Sammut, S.; Koh, R.G.L.; Zariffa, J. Compensation Strategies for Bioelectric Signal Changes in Chronic Selective Nerve Cuff Recordings: A Simulation Study. Sensors 2021, 21, 506 https://doi.org/10.3390/s210205 06

Received: 23 November 2020 Accepted: 8 January 2021 Published: 12 January 2021

Publisher's Note: MDPI stays neutral with regard to jurisdictional clai$\mathrm{ms}$ in published maps and institutional affiliations.

Copyright: (C) 2021 by the authors. Licensee MDPI, Basel, Switzerland. This article is an open access article distributed under the terms and conditions of the Creative Commons Attribution (CC BY) license (https:// creativecommons.org/licenses/by/ $4.0 /)$.

\begin{abstract}
Peripheral nerve interfaces (PNIs) allow us to extract motor, sensory, and autonomic information from the nervous system and use it as control signals in neuroprosthetic and neuromodulation applications. Recent efforts have aimed to improve the recording selectivity of PNIs, including by using spatiotemporal patterns from multi-contact nerve cuff electrodes as input to a convolutional neural network (CNN). Before such a methodology can be translated to humans, its performance in chronic implantation scenarios must be evaluated. In this simulation study, approaches were evaluated for maintaining selective recording performance in the presence of two chronic implantation challenges: the growth of encapsulation tissue and rotation of the nerve cuff electrode. Performance over time was examined in three conditions: training the $\mathrm{CNN}$ at baseline only, supervised re-training with explicitly labeled data at periodic intervals, and a semi-supervised self-learning approach. This study demonstrated that a selective recording algorithm trained at baseline will likely fail over time due to changes in signal characteristics resulting from the chronic challenges. Results further showed that periodically recalibrating the selective recording algorithm could maintain its performance over time, and that a self-learning approach has the potential to reduce the frequency of recalibration.
\end{abstract}

Keywords: neural interfaces; neural recording; peripheral nerve; nerve cuff electrode; chronic implantation; selective recording; machine learning; self-learning; encapsulation tissue

\section{Introduction}

The peripheral nervous system controls the flow of motor, sensory, and autonomic information between the central nervous system and other parts of the body, therefore mediating control over a multitude of physiological functions. Bioelectric signals recorded from the nervous system can be used to create commands for neuroprosthetic devices (which interact with the nervous system to restore lost function) and to regulate the activity of the nervous system through closed-loop neuromodulation. Examples of peripheral nerve recordings in neuroprosthetic applications include decoding motor signals to control a computer cursor [1,2], control a prosthetic limb [3,4], and using sensory feedback to control a functional electrical stimulation system [5,6]. Examples in neuromodulation applications include regulating blood pressure through selective vagus nerve stimulation [7] and using the electroneurogram of the vagus nerve to detect the onset of a seizure $[8,9]$. Currently, a major roadblock to closed-loop neuroprosthetic and neuromodulation applications is the need for techniques that can extract useful information from low-amplitude, noisy recordings [10].

Of the available peripheral nerve electrode designs [11], nerve cuff electrodes are an appealing choice as they have a relatively long history of use [12-14] and have been shown 
to be safe for long-term or chronic implantation in both animals [15-21] and humans [6,22]. However, these devices are extraneural (i.e., do not penetrate the nerve), resulting in a low signal-to-noise ratio (SNR) and recording selectivity.

As such, a variety of strategies have been developed in order to improve upon the selectivity and performance of these interfaces, e.g., cuff electrodes with multiple sites [23-26], intricate stimulation modes $[23,27]$, and the implementation of various signal processing algorithms $[28,29]$. Several research groups have attempted to improve the recording selectivity of cuff electrodes by using multi-contact nerve cuff configurations. For example, conduction velocity information has been determined by investigating the propagation of the neural signal across multiple contacts along the length of the nerve [30-34]. Alternatively, a number of studies have attempted to achieve the spatial selectivity of neural recordings using beamforming [15,35-37] or source localization methods [38,39]. Building on the results above, Koh et al. used spatiotemporal signatures to characterize the recordings from a nerve cuff with an array of contacts [40-42]. By using these spatiotemporal signatures as input to a convolutional neural network (CNN), individual natural compound action potentials (nCAPs) could be classified according to the neural pathway that produced them [42]. This CNN is referred to as the extraneural spatiotemporal compound action potential extraction network, or ESCAPE-NET. In this manner, the firing patterns of different pathways could be reconstructed, thus improving recording selectivity compared to previous approaches.

Though ESCAPE-NET has achieved promising results in acute experiments, its response to the functional and morphological changes that occur during chronic implantation must be investigated before it can be translated to human subjects. Here, we used a simulation study to characterize the changes in the performance of the selective recording algorithm over time and to evaluate strategies to compensate for these changes. In this study, two major factors likely to cause bioelectric signal changes in the recordings of multi-contact nerve cuff electrodes, and therefore reduce selectivity, were simulated: (1) the buildup of encapsulation tissue surrounding the nerve, which is an immune response to an implanted neural electrode that causes changes in the electrical interface [43-45], and (2) shifts in the position of the nerve cuff electrode as a result of physical movement, which are known to impact recording selectivity [46,47]. After simulating and characterizing the changes that occurred over time, we compared three approaches for updating ESCAPE-NET during chronic implantation in order to maintain high levels of performance.

\section{Methods}

The first step in our study was to simulate recordings from a multi-contact nerve cuff electrode implanted on a rat sciatic nerve. We then altered the model to simulate signal changes during chronic implantations. Lastly, methods to compensate for those changes over time were compared.

\subsection{Model Construction}

A previously described finite element model of the rat sciatic nerve and nerve cuff electrode in a saline bath [46] was used as the basis for our simulations in this study. The model was originally developed using magnetic resonance imaging of the nerve and includes progressive branching of the sciatic nerve into the tibial, peroneal, and sural nerves. The anatomy of this model was manually segmented using Seg3D [48], before implementing the modifications described below.

The endoneurium and epineurium were manually traced and thus accurately reflected the physical dimensions of these structures, while the perineurium was added around each fascicle with an approximate thickness of $0.065 \mathrm{~mm}$, due to the fact that thinner layers have previously been found to cause errors in the meshing process [46].

Once the segmentation of the rat sciatic nerve was completed and verified, it was exported to MATLAB, where a tetrahedral mesh model was generated using Iso2Mesh [49]. 
This baseline is shown in Figure 1. The layout of the nerve cuff electrode is shown in Figure 2.
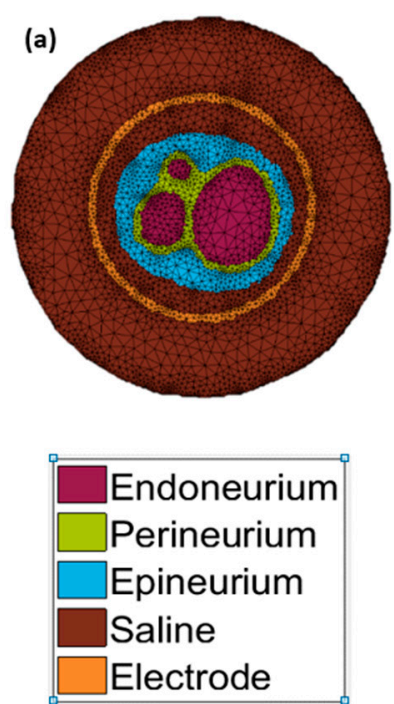

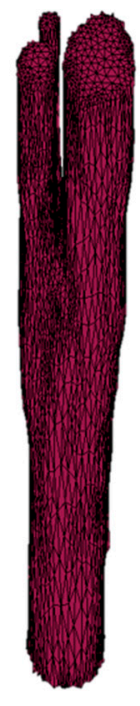

(b)

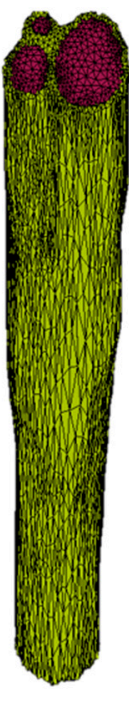

(c)

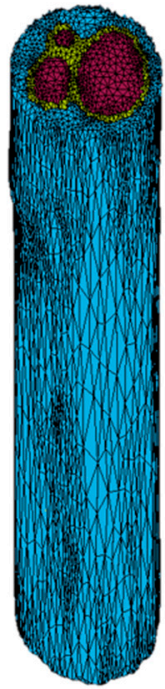

(d)

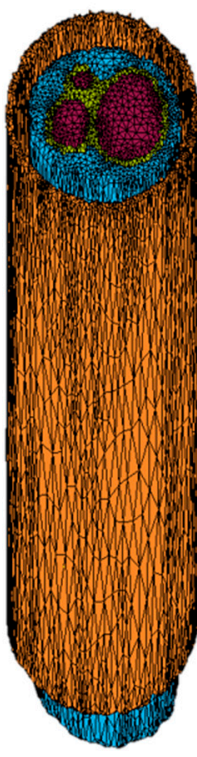

(e)

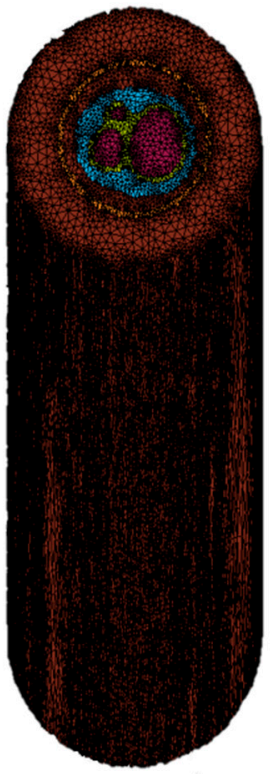

(f)

Figure 1. Finite element (FE) tetrahedral mesh model. (a) Top-down view of the base FE mesh model. Individual layers of the FE mesh model: (b) endoneurium, (c) perineurium, (d) epineurium, (e) cuff electrode, and (f) saline.

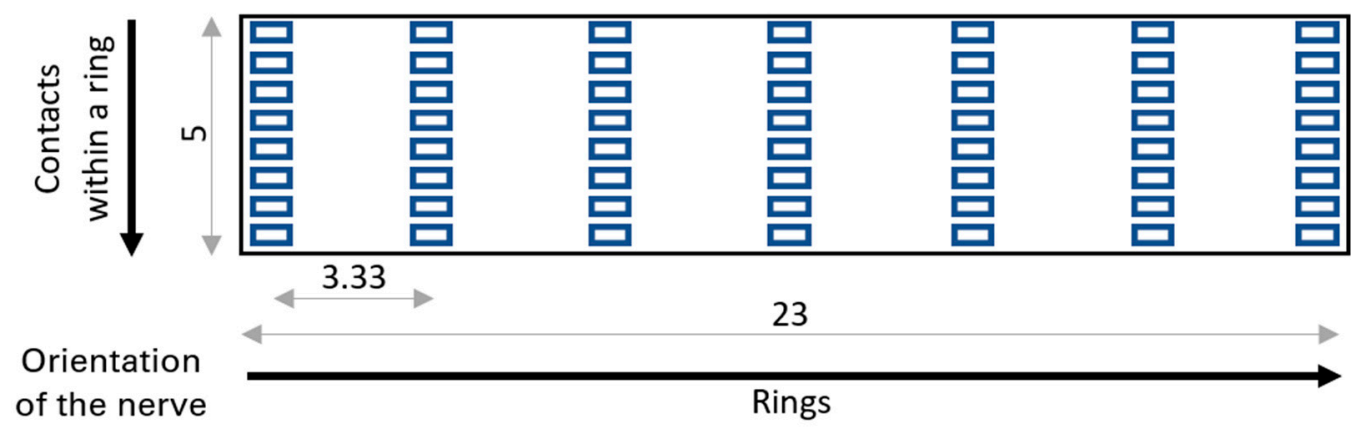

Figure 2. Visualization of the unrolled nerve cuff electrode design used in modelling, with dimensions shown (millimeters).

Following the generation of the mesh model, finite element analysis was conducted in SCIRun [50] in order to generate a leadfield matrix. The leadfield, represented by a matrix $\mathbf{L}$ with entries $(i, j)$, characterized the influence that a unit current dipole positioned at a mesh element $j$ had on the electrical potential observed at an electrode contact $i$. $\mathbf{L}$ was of size $\mathrm{M} \times \mathrm{N}$, where $\mathrm{M}$ represents the number of electrode contacts and $\mathrm{N}$ represents the number of elements that form the tetrahedral mesh. The leadfield matrix was constructed according to the process described by Weinstein et al. [51].

Parameters of the tetrahedral mesh model, including conductivity values and structure dimensions, are summarized in Table 1, and were established based on similar models described in the literature. 
Table 1. FE mesh model parameters.

\begin{tabular}{ccc}
\hline Parameter & Value & References \\
\hline Nerve Length & $26 \mathrm{~mm}$ & {$[46]$} \\
\hline Cuff Length & $23 \mathrm{~mm}$ & {$[46]$} \\
\hline Cuff Width & $60 \mu \mathrm{m}$ & {$[46]$} \\
\hline Cuff Radius & $800 \mu \mathrm{m}$ & {$[46]$} \\
\hline Endoneurium conductivity (radial) & $8.26 \times 10^{-2} \mathrm{~S} \mathrm{~m}^{-1}$ & {$[38,46,52]$} \\
\hline Endoneurium conductivity (longitudinal) & $5.71 \times 10^{-1} \mathrm{~S} \mathrm{~m}^{-1}$ & {$[38,46,52]$} \\
\hline Perineurium conductivity (all directions) & $2.10 \times 10^{-3} \mathrm{~S} \mathrm{~m}^{-1}$ & {$[38,46,52]$} \\
\hline Epineurium conductivity (all directions) & $8.26 \times 10^{-2} \mathrm{~S} \mathrm{~m}^{-1}$ & {$[38,46,52]$} \\
\hline Encapsulation tissue conductivity (all directions) & $6.59 \times 10^{-2} \mathrm{~S} \mathrm{~m}^{-1}$ & {$[38,53]$} \\
\hline Saline Conductivity (all directions) & $2.00 \times 10^{-1} \mathrm{~S} \mathrm{~m}^{-1}$ & {$[38,46,52]$} \\
\hline Cuff Conductivity (all directions) & $1.00 \times 10^{-7} \mathrm{~S} \mathrm{~m}^{-1}$ & {$[38,46,52]$} \\
\hline
\end{tabular}

\subsection{Simulation of Chronic Factors}

\subsubsection{Growth of Encapsulation Tissue}

The original model was modified in order to incorporate encapsulating tissue into the segmentation as an additional material type. This new structure was incorporated into the original segmentation between the epineurium and cuff electrode throughout each layer, as illustrated in Figure 3. This process was repeated multiple times in order to generate a total of four models with varying degrees of encapsulation tissue growth (referred to as ET1, ET2, etc.) and therefore simulate a number of time points following the implantation of the nerve cuff electrode. In this simulation, time elapsing was simulated through the continuous growth of encapsulation tissue between models. Note that in reality, encapsulation tissue will eventually also surround the cuff. However, the external layer of tissue was unlikely to affect our simulated measurements at the recording contacts, which are located on the inner surface of the cuff, and therefore was neglected in our modeling.

\subsubsection{Rotation of the Nerve Cuff Electrode}

In order to simulate the rotation of the nerve cuff electrode around the rat sciatic nerve, the electrode positions were rotated in three-dimensional space around the central axis of the nerve. Given the geometry of this nerve cuff electrode, eight contacts in each ring surround the perimeter of the nerve. As such, a rotation of $45^{\circ}\left(360^{\circ} / 8\right.$ contacts $\left.=45^{\circ}\right)$ from the original position resulted in all electrode contacts being placed in the starting position of a different contact. For this reason, the $45^{\circ}$ was selected as the maximal rotation, and increments of $5^{\circ}$ were simulated. A new leadfield was generated for each rotation using the new contact locations. In this simulation, deviation from the original signals was simulated through continuous rotation of the nerve cuff electrode.

\subsection{Simulated Recordings}

Myelinated mammalian fiber action potentials were obtained with the Neurocal MATLAB toolbox (Case Western University, Cleveland, OH, USA) using the Schwarz-ReidBostock model [54]. This model is typically used to simulate single fiber action potentials; however, a simplifying assumption was made here that it could be used to approximate the shape of a CAP; this assumption corresponded to the simplified CAP scenario of multiple nerve fibers firing in perfect synchrony, leading to a version of the single fiber action potential with scaled-up amplitude, as used in previous modeling [40]. 


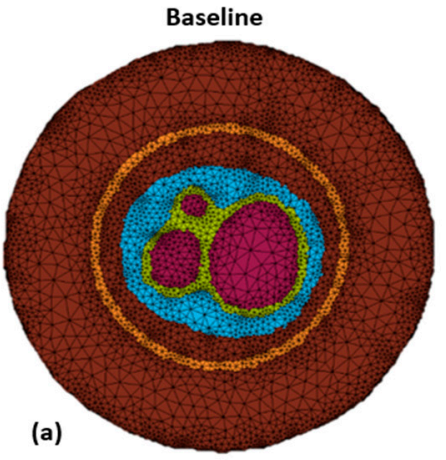

ET2

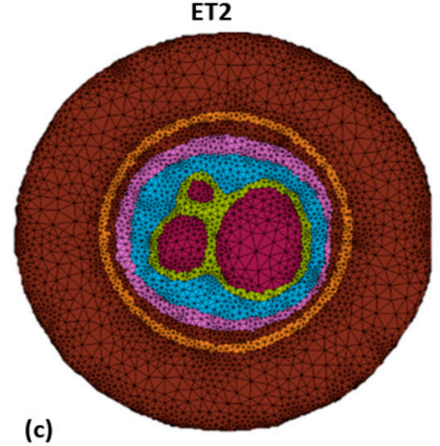

(d)

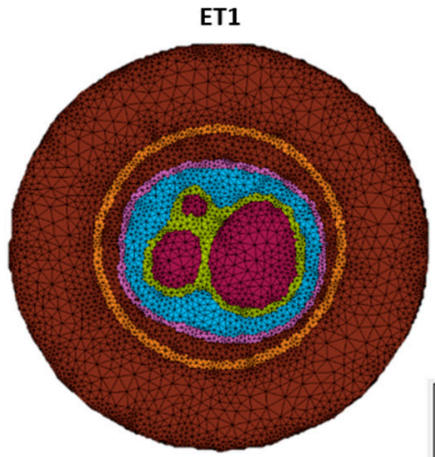

ET3

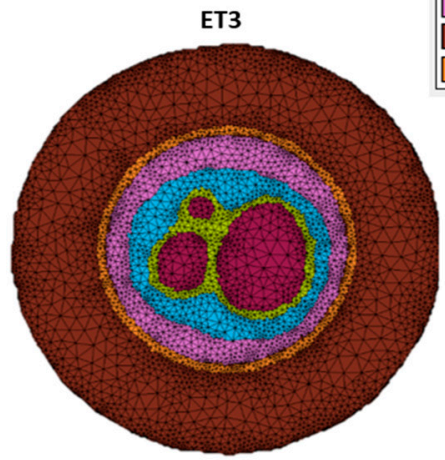

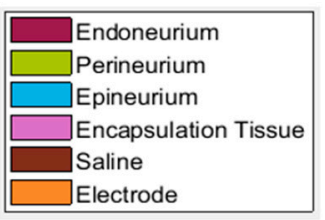

Figure 3. Top-down view of FE mesh models from: (a-d) no encapsulation tissue to the full encapsulation of the nerve.

The CAPs were propagated through the model at differing spatial locations within each of three neural pathways by selecting mesh elements from within a given fascicle. Multiple points for a single pathway were manually defined throughout the model starting from the proximal end and moving towards the distal end. MATLAB was used in order to generate a spline interpolation along the entire length of the nerve from the provided points, thus defining a neural pathway [46]. This procedure was performed for the tibial, peroneal, and sural fascicles.

The positioning of the Nodes of Ranvier along a neural pathway was defined according to the fiber type being simulated. In this simulation, the spacing of the Nodes Ranvier were $1.70 \mathrm{~mm}$ for $\mathrm{A} \alpha$ fibers (tibial and peroneal pathways) and $1.16 \mathrm{~mm}$ for $\mathrm{A} \beta$ fibers (sural pathway). Conduction velocities of 94.86 and $64.72 \mathrm{~m} / \mathrm{s}$ were used for the $\mathrm{A} \alpha$ and A $\beta$ fibers, respectively [40]. These parameters were used in conjunction with the leadfield matrix to simulate all 56 channel measurements of a CAP propagating through a single neural pathway. The simulated recordings were then tripole-referenced using the average of all outer ring contacts [55].

By combining CAPs from a single neural pathway, a time series was created. A single time series was created for each of the three fascicles (tibial, peroneal, and sural), composed of 10,000 CAPs within a duration of 53.333 seconds. These specifications were chosen in order to add noise to the simulated recordings in a consistent manner to Koh et al. [40]; hereafter, CAPs were analyzed individually without consideration of firing rates. White Gaussian noise was then added to the time series in order to simulate baseline and instrumentation noise commonly observed in these types of recordings in vivo by using decreasing levels of signal-to-noise ratios $(-5,-10$, and $-15 \mathrm{~dB})$, defined using the measured signal power over the entire time series. 


\subsection{CAP Classification}

\subsubsection{Datasets}

The simulated CAPs were individually classified according to the neural pathway used to generate the recording (tibial, peroneal, or sural fascicle). Therefore, classification here was a three-class problem.

Once the noisy time series were created, individual CAP spatiotemporal signatures were extracted based on peak detection prior to the addition of noise. Signatures that were 100 time samples long ( $3.333 \mathrm{~ms}$, using a sampling rate of $30 \mathrm{kHz}$ ) were created by obtaining 49 points before and 50 points after the location of the CAP peak for each of the 56 channels. These data were then normalized within the range from -1 to 1 based on the maximum amplitude seen across all CAPs. These data points served as either training or testing samples, depending on the strategy being evaluated, and were provided to our CNN (ESCAPE-NET) with their respective labels based on the neural pathway producing them. A dataset containing a total of 30,000 CAPs $(10,000$ CAPs $\times 3$ pathways) was created for each model in each of the two simulations (encapsulation tissue growth and rotation of the cuff electrode).

\subsubsection{Convolutional Neural Network}

A previously described CNN, ESCAPE-NET [42], was used to classify the spatiotemporal signatures produced by the CAPs in the multi-contact nerve cuff. As such, the inputs to ESCAPE-NET were $56 \times 100$ spatiotemporal signatures created as described above. The CNN used in this work was constructed with Python 2.7 using the Keras application programming interface (API) with TensorFlow [56] as the backend.

The spatiotemporal signature used as input to ESCAPE-NET can exhibit different patterns depending on the ordering of the contacts. Alternatively, the network can be provided with more than one such representation, in order to exploit possibly complimentary information [42]. In the case of this project, ESCAPE-NET's dual input version was used. In this version of the network, the architecture is split into two identical sides in order to process two input configurations obtained from the matrix of the multi-contact nerve cuff electrode recordings (contacts ordered by rings or longitudinally, referred to as "spatial emphasis" or "temporal emphasis", respectively). A block diagram of ESCAPE-NET's architecture can be seen in Figure 4, while additional details can be found in [42].

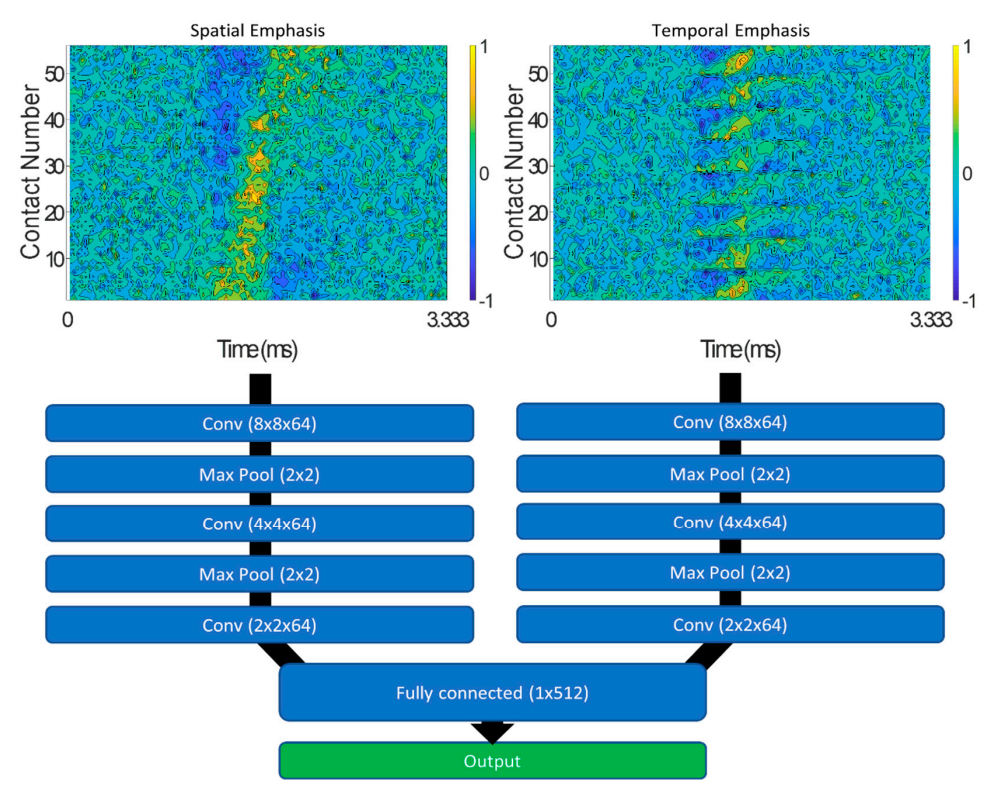

Figure 4. ESCAPE-NET (extraneural spatiotemporal compound action potential extraction network) architecture with spatial and temporal emphasis representations of the multi-contact nerve cuff recordings (signal-to-noise ratio $(\mathrm{SNR})=-5 \mathrm{~dB}$ ) shown as input to the network [42]. 


\subsection{Classifier Update Strategies}

When applying the $\mathrm{CNN}$ over the multiple simulated time points, one of three training approaches was used: (1) baseline calibration (control condition), (2) periodic recalibration, and (3) a self-learning approach.

Evaluation was achieved in all three of the approaches using five-fold cross validation. In order to create a training and testing set for the convolutional neural network, the 30,000 CAPs generated for each model were split into five folds, with each fold containing 6000 CAPs (2000 CAPs from each of the three pathways). Each fold was used in turn as the testing set for cross-validation in order to evaluate each of the strategies at each time point. The analysis was repeated separately for each of the 3 noise levels. The classification performance of each network at each time point was assessed by calculating the macro $\mathrm{F}_{1}$-score (i.e., calculating the $\mathrm{F}_{1}$-score for each class and averaging them).

\subsubsection{Baseline Calibration}

In the first approach, the classifier was trained once, at baseline, representing the initial implantation of the nerve cuff electrode with no encapsulation tissue or rotation present. The CNN was evaluated on data from subsequent time points without further modification. In this way, we quantified the performance of the classifier over time as factors likely to cause bioelectrical signal drift begin to arise during chronic implantation. This simulation represented a scenario in which a neural interface is calibrated at the time of initial implantation, and its performance was evaluated over time without additional recalibration.

\subsubsection{Periodic Recalibration}

In the second approach, the network, again initially trained at baseline, was retrained at future points in time with new, explicitly labelled data. At each new time point, the network was first initialized with the final learned weights of the previous time point, then retrained using an explicitly labelled datasets generated by the model for the current time point. This process was repeated for each subsequent point in time. This simulation represented a scenario in which a user carries out an explicit recalibration procedure at regular intervals.

To evaluate the periodic calibration approach, the supervised network was retrained at each new time point with four folds (24,000 CAPs) of the new time point data, and then it was tested using the remaining fold (6000 CAPs). This process was repeated using each of the five cross-validation folds in turn as the test set.

\subsubsection{Self-Learning Approach}

In the final approach, the network, again initially trained at baseline, was retrained at future time points without the need for the manual labelling of new data. This approach was based on methods described by Schwemmer et al. [57] and utilized "predicted" data. At each new time point, new data were first classified using the network obtained in the previous time point. Only data samples predicted by the network with high-enough confidence ( $95 \%$ confidence) were chosen, along with their estimated labels, in order to create what is referred to as a "self-labelled" dataset (see Figure 5). The network was then initialized with the final learned weights of the previous time point and retrained using the self-labelled dataset in order to update its weights. This process was repeated for each subsequent point in time. This simulation represented a scenario in which an implanted neural interface is recalibrated periodically without the need for user or experimenter intervention.

To evaluate the self-learning approach, the network at each new time point was first initialized using the learned weights from the previous time point. The classifier was retrained with four folds of the new time point data, labelled by the network itself. The network was then tested using the remaining fold but with the explicit labels provided. The process was repeated five times with different testing folds for cross validation. 


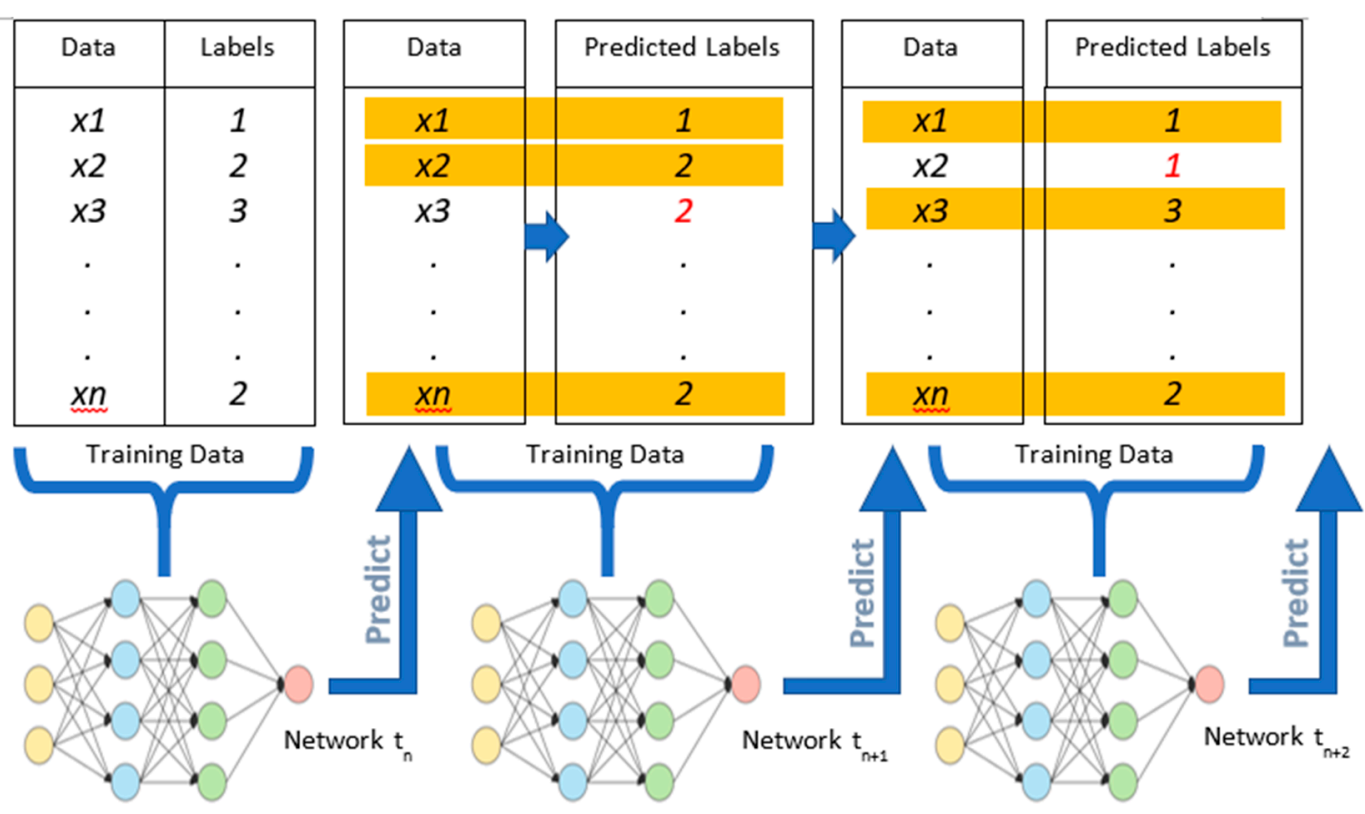

Figure 5. Visual representation of the processes involved in the self-learning approach. In each step, the previously obtained model is used to predict labels at the new time point. The network is then updated/retrained using these predicted datasets, at the current point in time. Here, the highlighted text represents correctly predicted samples, while red text represents mislabeled samples, both of which may be incorporated into a "self-labelled" dataset depending on the confidence level.

The classification of the network that was achieved using each of the three updating strategies was assessed by calculating the macro $F_{1}$-score (i.e., calculating the $F_{1}$-score of each class and then averaging them).

A timeline of the training and testing of the $\mathrm{CNN}$ involved in each of the three update strategies, using the growth of encapsulation tissue simulation as an example, is depicted below in Figure 6.

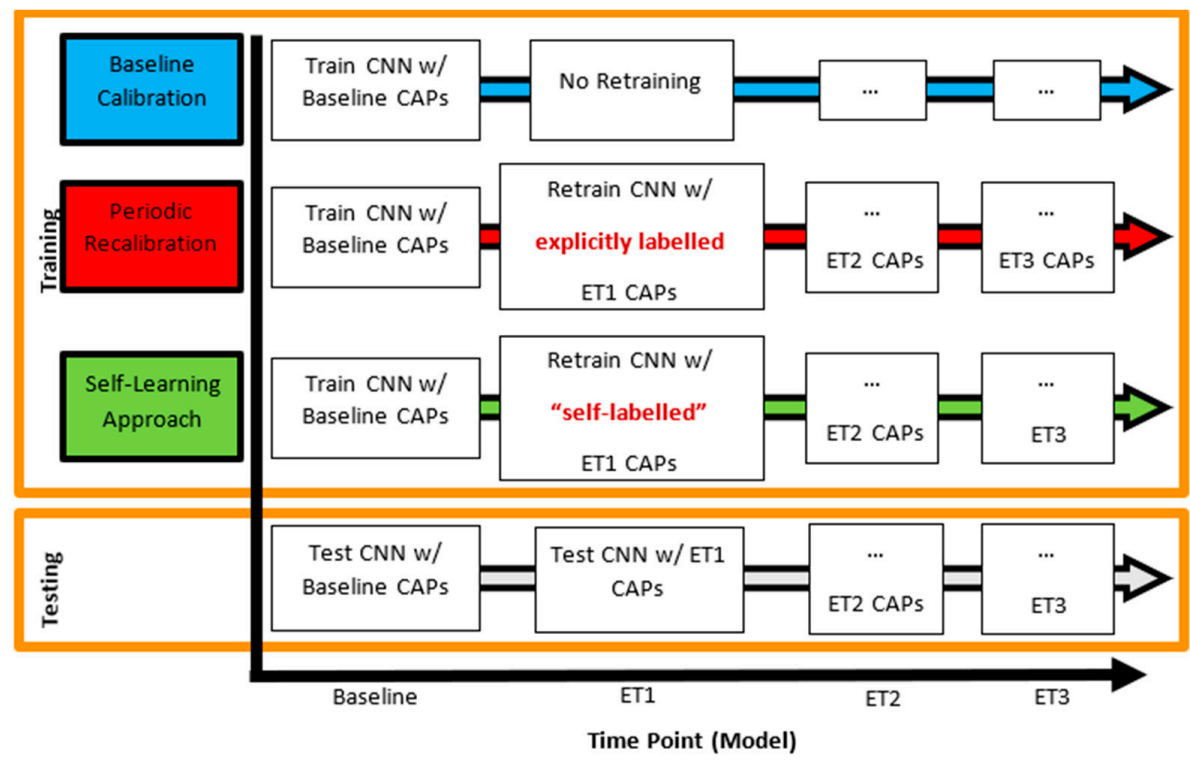

Figure 6. Training and testing scenarios of the three updating approaches. The vertical axis of the figure shows the update strategy, while the horizontal axis represents time elapsing and subsequent changes in the interface. Text boxes within the figure show training and testing approaches corresponding to specific update strategies at a given time point (in this case representing the growth of encapsulation tissue). 


\subsubsection{Further Analysis of the Self-Learning Approach}

Due to the nature of the self-learning approach, several factors are likely to influence the efficacy of this machine learning update strategy. We conducted additional analyses of the encapsulation tissue simulation to characterize the impact of the following parameters.

\section{Training Frequency}

First, we hypothesized that the greater the time between training intervals, the less effective the self-learning method would be. This hypothesis was based on the assumption that a relatively long interval between training times would correspond to greater changes in the recorded signals. Large signal changes would lead to the poorer performance of a classifier trained at the previous time point, which in turn may cause inaccurate predicted labels and a degradation in classification accuracy. As such, updating the network at less frequent time points would likely result in a poorer performance over time. We therefore investigated the efficacy of the self-learning approach in scenarios where the time between training intervals was modified.

In order to investigate whether the self-learning approach would benefit from more frequent training of the $\mathrm{CNN}$, linear interpolation was used between the CAPs from successive time points in the finite element models in order to generate data from additional time points. This approximation was chosen because it would be quite difficult to create additional three-dimensional mesh models with smaller, more gradual increases in encapsulation tissue growth due to space limitations in the segmentation step and the need for finer meshes. In this experiment, the self-learning approach was implemented as described above, and the network was then retrained twice, four-times, and eight-times as often as the other two update strategies.

\section{Initial Performance Level}

Another factor that could affect the efficacy of the self-learning approach is the initial performance level. If the performance of the network from the previous time point is relatively high, it may more correctly predict and thus label samples in the new time point. Conversely, an inaccurate network could predict incorrect labels, leading to error accumulation and performance degradation. As such, the performance of the network at one time point will affect the number and accuracy of the labelled samples at the next time point. We therefore investigated the efficacy of the self-learning approach in scenarios where the initial performance level was modified.

To this end, we needed a set of different initial performance levels. This was accomplished using the results achieved at ET1 for different updating methods and SNRs. Thus, for this portion of the analysis, ET1 was used as the starting point. Specifically, six different initial starting performances were achieved by initializing the network with weights obtained at baseline at each noise level $(-5,-10$, and $-15 \mathrm{~dB})$ and then applying either the periodic recalibration approach or the self-learning approach at ET1.

\section{Evaluation}

Different training frequencies $(1 \times, 2 \times, 4 \times$, and $8 \times)$ were applied for each starting level at each SNR. The purpose of this analysis was to understand how the self-learning approach performed compared to a no-update control, depending on the initial performance and retraining frequency. The control was a model trained at ET1 for each of the initial performance levels and applied to subsequent time points without retraining.

The primary metric for this analysis was the slope of the $\mathrm{F}_{1}$-score between ET1 and ET2 compared to the slope of the control. For example, a method that led to a higher performance than the control at ET2 would have a less negative slope and could thus be said to be beneficial, i.e., to have slowed the decline in performance over time. 


\section{Results}

\subsection{Simulated Recordings}

Once a compound action potential had been propagated through the model, measurements at all 56 channels of the simulated nerve cuff electrode were obtained, as illustrated in Figure 7a (shown with no noise added). Examples of time series segments with different noise levels are shown in Figure $7 \mathrm{~b}$.

(a)

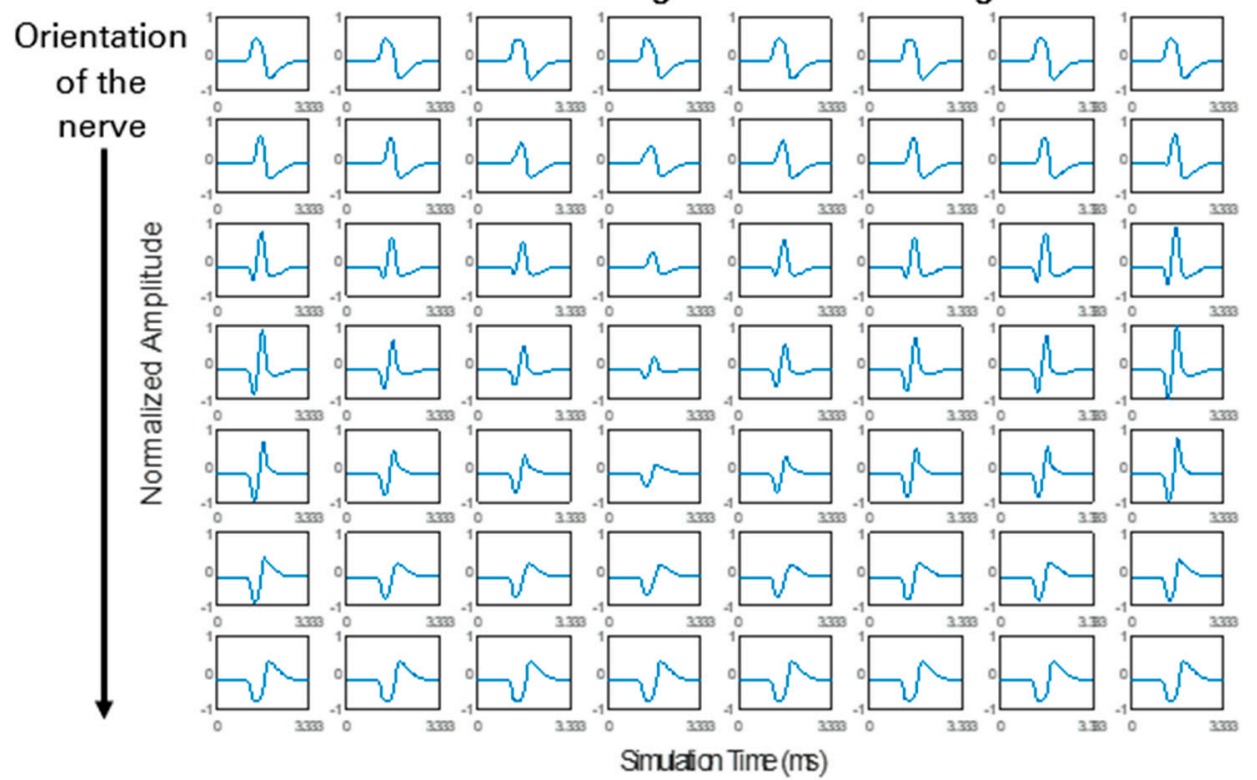

(b)

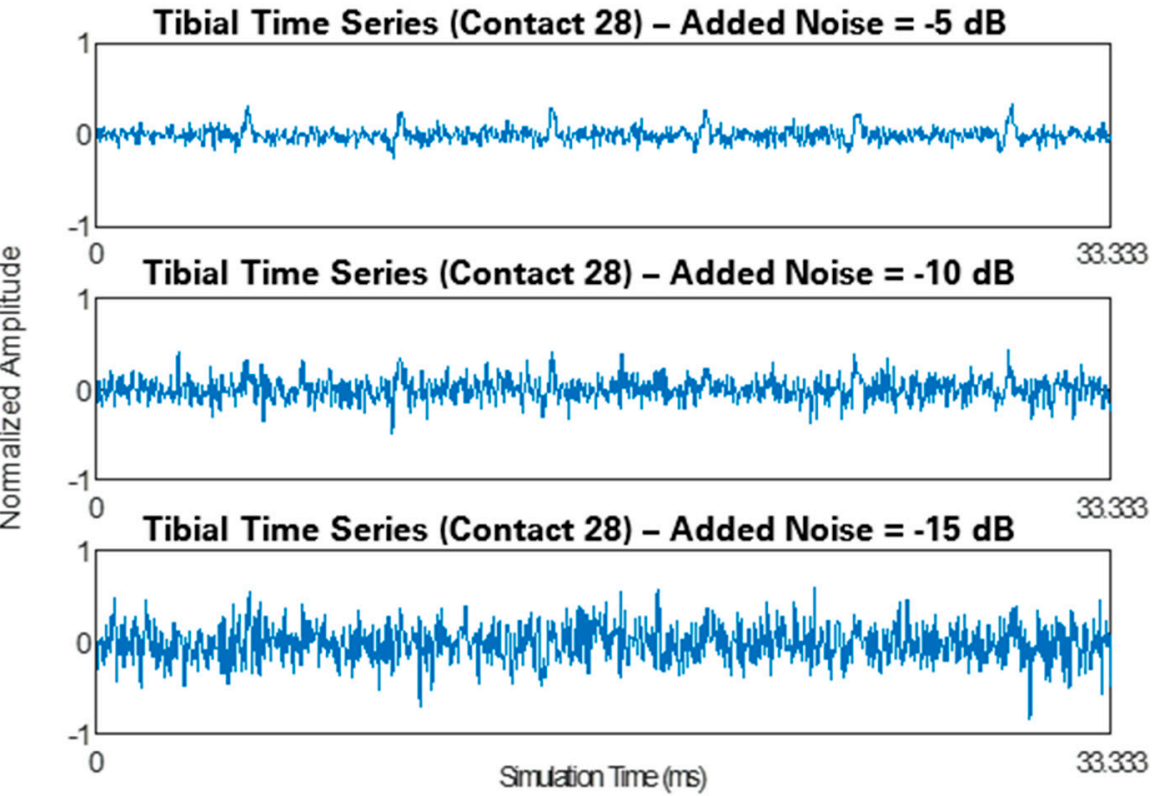

Figure 7. (a) Simulated recordings at all 56 contacts of the cuff electrode for a single compound action potential (CAP) propagated through the tibial pathway. (b) Segments of the tibial time series measurements of the middle contact (contact 28 ) at different noise levels ( $\mathrm{SNR}=-5,-10$, and $-15 \mathrm{~dB}$ ). All data here have been normalized for visualization purposes.

\subsection{Classification}

\subsubsection{Growth of Encapsulation Tissue}

Figure 8 shows the mean $\mathrm{F}_{1}$-score obtained by the $\mathrm{CNN}$ at each time point in the encapsulation tissue growth simulation using the three updating strategies at decreasing 
levels of the SNR. The baseline calibration approach, in which the performance of a static $\mathrm{CNN}$ was evaluated at specific time points, began to degrade as the encapsulation tissue grew. This trend was more evident at higher SNRs of -5 and $-10 \mathrm{~dB}$, whereas the performance of the baseline calibration approach was relatively more stable at the lowest $\mathrm{SNR}$ of $-15 \mathrm{~dB}$. In contrast, the periodic recalibration approach led to an improvement in classification performance. The self-learning approach, in which the $\mathrm{CNN}$ was retrained with self-labelled data at specific time points, showed a degradation in classification performance over time, similar to that of the baseline calibration approach.

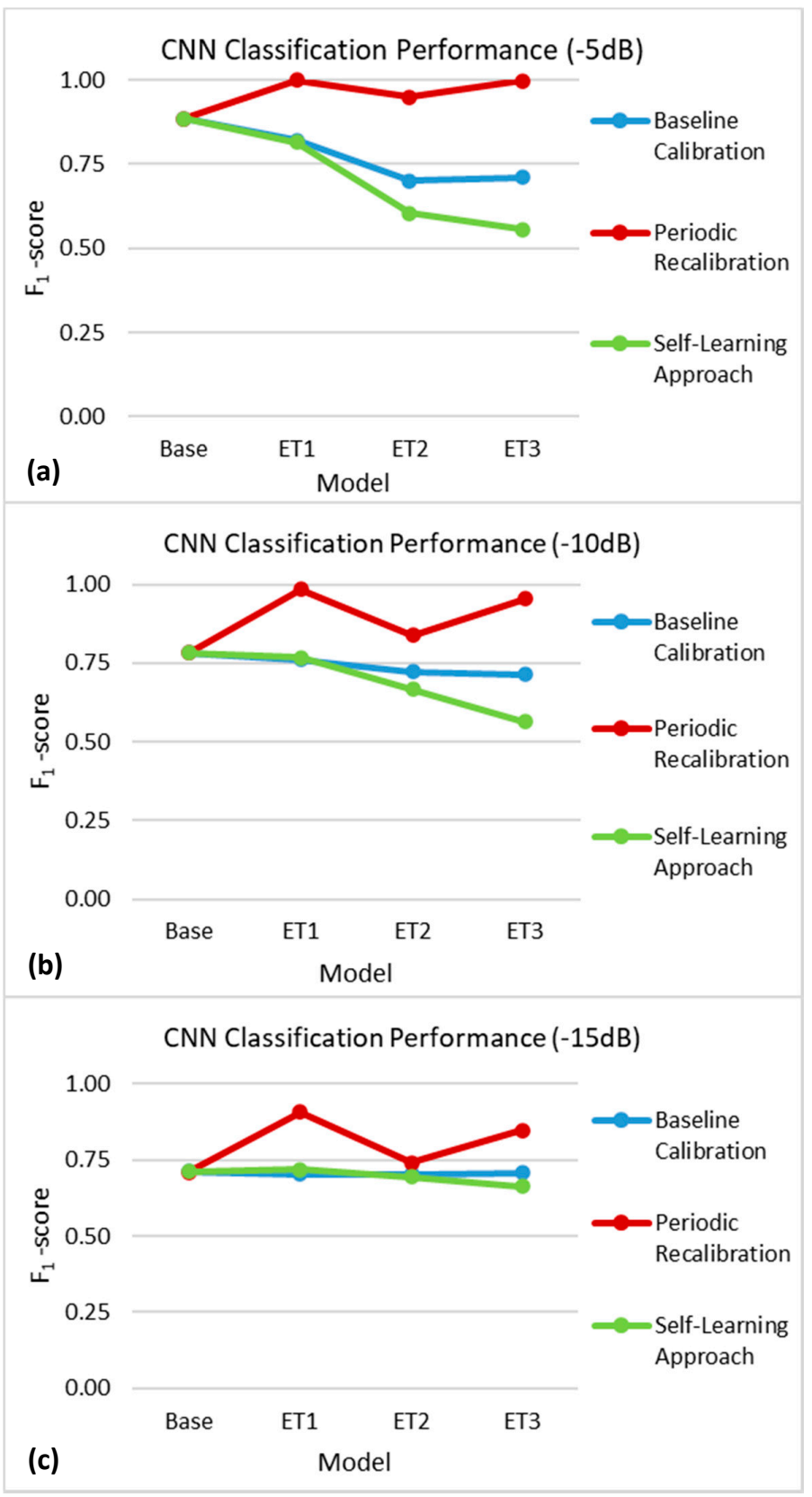

Figure 8. $\mathrm{F}_{1}$-scores obtained in the encapsulation tissue simulation using the baseline calibration (blue), periodic recalibration (red), and self-learning (green) update approaches at different time points (represented here as additional models) using measurements with (a) $-5 \mathrm{~dB},(\mathbf{b})-10 \mathrm{~dB}$, and (c) $-15 \mathrm{~dB}$ of noise added. The standard deviations at each time point were all below 0.007 and are therefore not displayed here for ease of visualization. 


\subsubsection{Rotation of the Nerve Cuff Electrode}

Figure 9 shows the mean $\mathrm{F}_{1}$-score obtained by the $\mathrm{CNN}$ at each time point in the rotation of the nerve cuff electrode simulation using the three updating strategies at decreasing levels of SNRs. The results for the baseline calibration approach were consistent with those of Figure 8, with a degradation of the selective recording performance as the rotation increased being most evident at the higher SNRs of -5 and $-10 \mathrm{~dB}$. In this scenario, the periodic recalibration approach led to a maintenance in classification performance. Once again, the self-learning approach led to a degradation in performance over time.

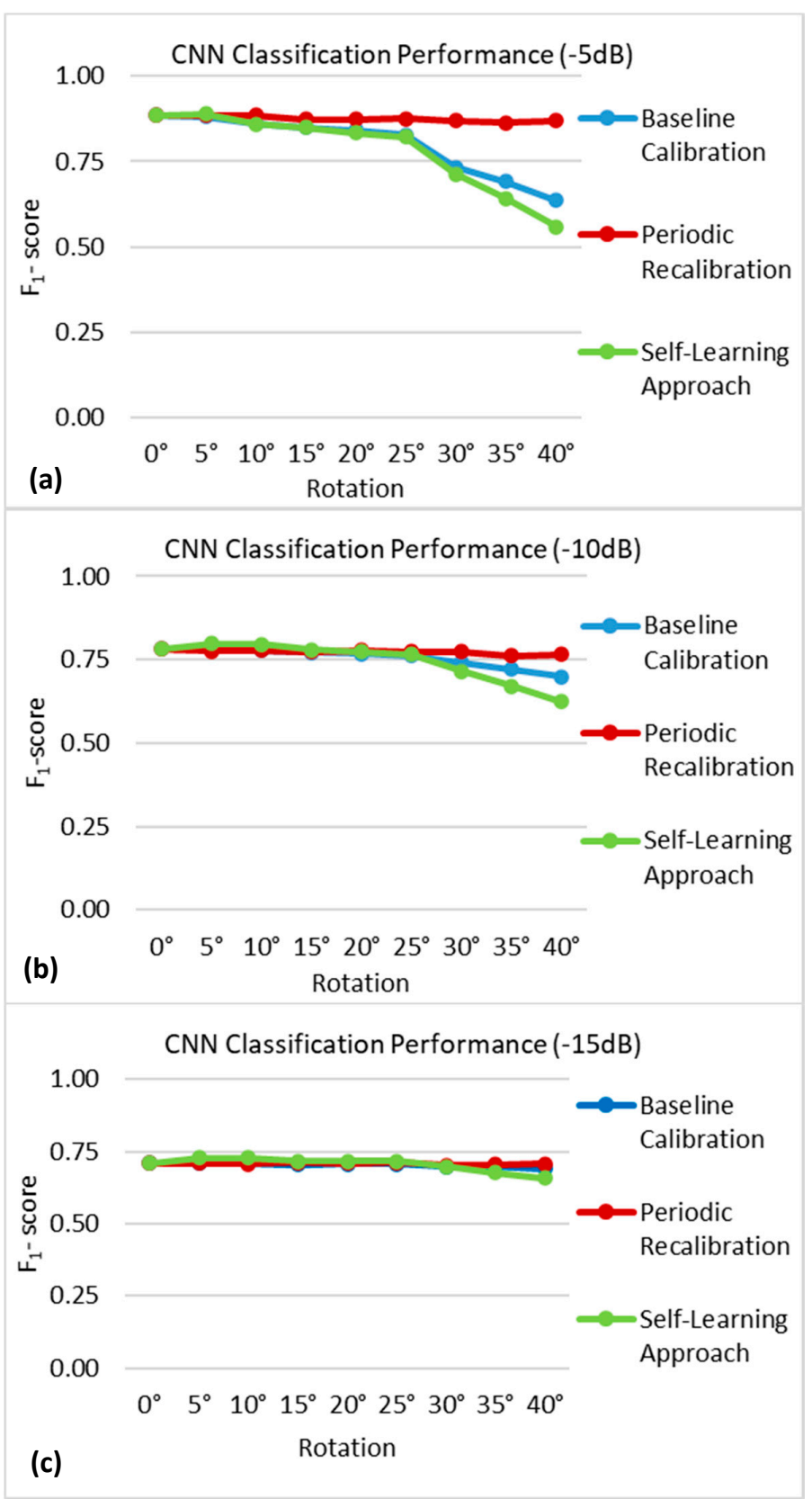

Figure 9. $\mathrm{F}_{1}$-scores obtained in the rotation of the cuff electrode simulation using the baseline calibration (blue), periodic recalibration (red), and self-learning (green) update approaches at different time points (represented here as additional models) using measurements with (a) $-5 \mathrm{~dB},(\mathbf{b})-10 \mathrm{~dB}$, and (c) $-15 \mathrm{~dB}$ of noise added. The standard deviations at each time point were all below 0.008 and are therefore not displayed here for ease of visualization. 
3.2.3. Influence of Training Frequency and Initial Performance on Self-Learning Approach Performance

The classification performance over time of the self-learning approach using different training frequencies and starting at two different initial performance levels for each noise level is shown in Figure 10. In each case, training and evaluation were conducted up until the time point in which there were no longer enough samples (i.e., less than 200) from each class to form a sufficient validation set.

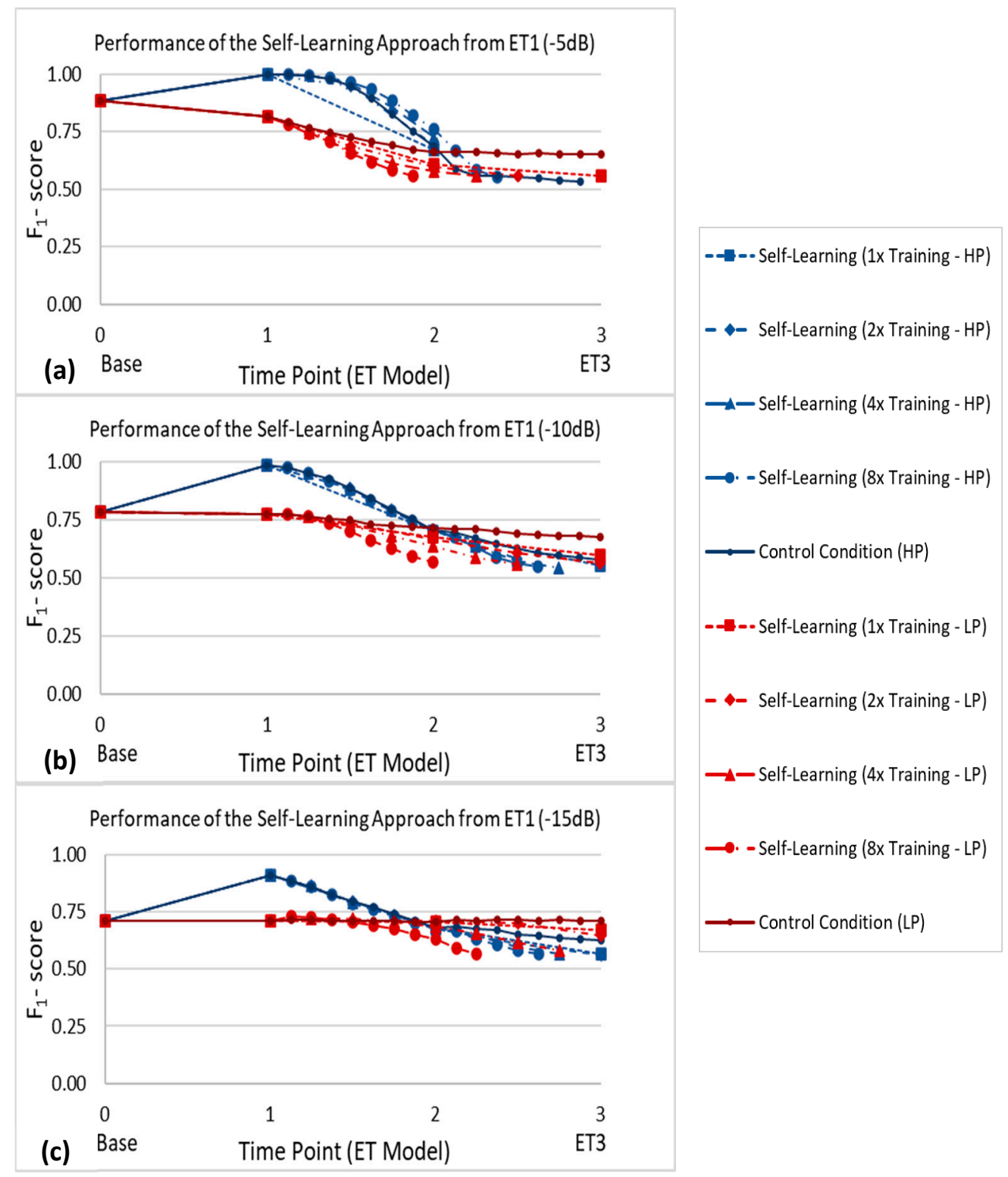

Figure 10. Classification performance of the self-learning approach using different training frequencies and starting at two different initial performance levels for SNRs of $(\mathbf{a})-5 \mathrm{~dB},(\mathbf{b})-10 \mathrm{~dB}$, and (c) $-15 \mathrm{~dB}$. Here, HP represents starting at the higher performance (HP) level shown as the blue curves, while LP represents starting at the lower performance (LP) level shown as the red curves. The starting point for HP was obtained by applying the periodic recalibration approach between Base and encapsulation tissue growth level 1 (ET1). The starting point for LP was obtained by applying the self-learning approach between Base and ET1. The standard deviations at each time point were all below 0.02 and are therefore not displayed here for ease of visualization.

Figure 11 shows how the rate of decline varied as a function of the training frequency, initial performance level, and noise level. The values represented on these plots were derived by subtracting the slope of the control between ET1 and ET2 from the corresponding 
slope of the self-learning classifier. Therefore, in Figure 11, red areas represent situations in which the self-learning approach outperformed the control condition, while blue areas represent situations in which the self-learning approach performed worse than the control condition.

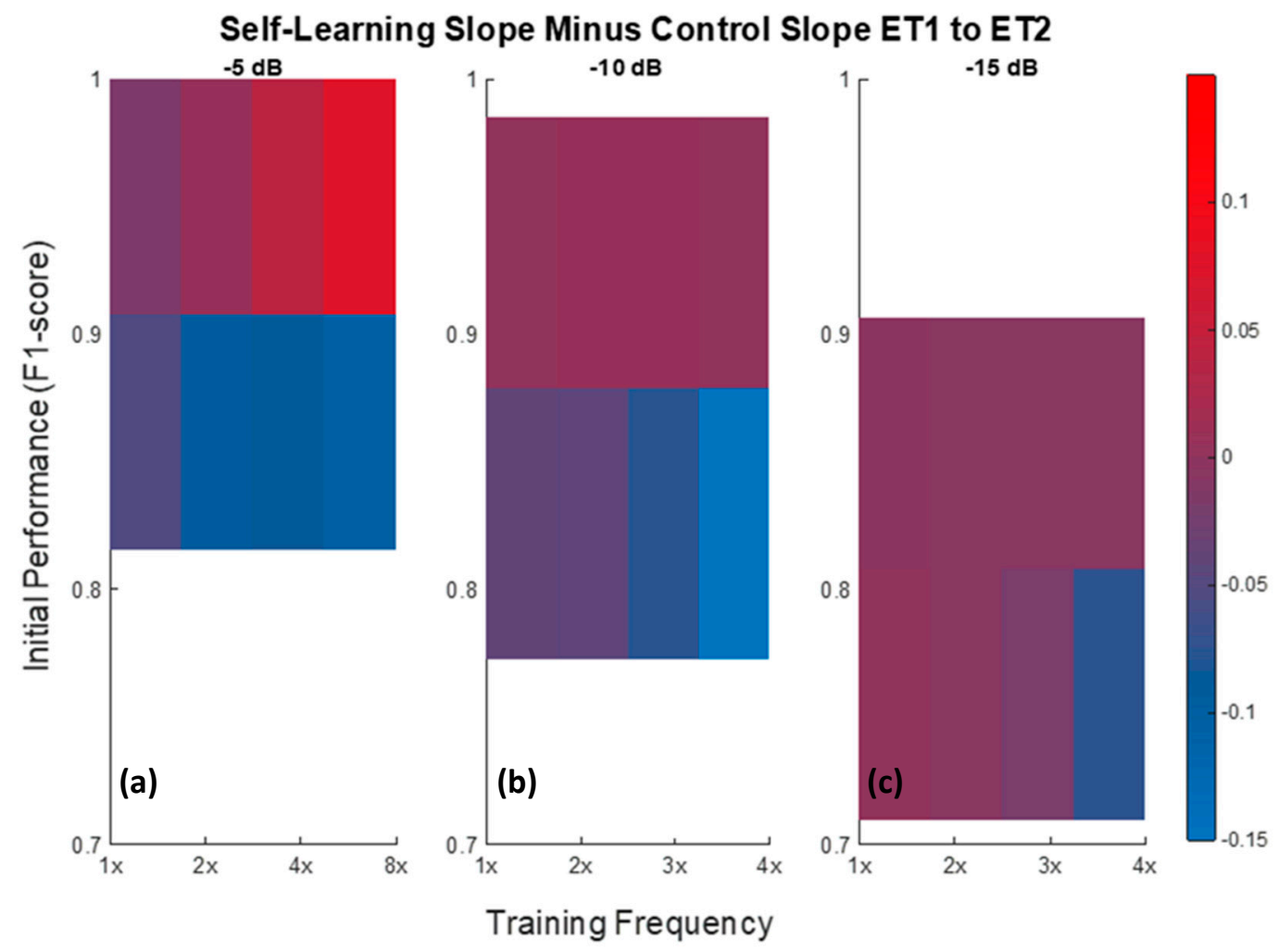

Figure 11. Self-learning slope minus control slope between ET1 and ET2 for SNRs of $-5,-10$, and $-15 \mathrm{~dB}$. The $-5 \mathrm{~dB}$ plot (a) shows two levels of initial performance $\left(\mathrm{F}_{1}\right.$-score $=0.9997$ and 0.8153$)$. The $-10 \mathrm{~dB}$ plot $(\mathbf{b})$ shows two levels of initial performance $\left(\mathrm{F}_{1}\right.$-score $=0.9852$ and 0.7720$)$. The $-15 \mathrm{~dB}$ plot $(\mathrm{c})$ shows two levels of initial performance $\left(\mathrm{F}_{1}\right.$-score $=0.9065$ and 0.7096).

\section{Discussion}

In this simulation study, we characterized the expected changes in the performance of a selective recording approach during chronic implantations and evaluated strategies to compensate for these changes. We focused on two commonly seen challenges in chronic peripheral nerve interface operation: the growth of encapsulation tissue and the movement of the nerve cuff electrode. In each of these scenarios, three update strategies were investigated: baseline calibration, periodic recalibration, and a self-learning approach The baseline calibration approach represented a control condition used to determine the effect of the particular challenge being investigated over time and therefore provided a comparison point for the other two update strategies.

\subsection{Baseline Calibration}

The degradation in classification performance over time when using baseline calibration in the encapsulation tissue simulation was expected, as previous research has shown that the growth of this encapsulating tissue is known to cause changes in the characteristics of the bioelectric signal [43-45]. Additionally, the results observed in the rotation of the nerve cuff simulation could be somewhat expected, as previous research has shown that a change in the initial position of the nerve cuff electrode will likely cause a modification in the bioelectric recordings due to the resultant change in the geometry of the volume conductor $[46,47,58]$. 
In summary, in both scenarios, we observed that a network initially trained at baseline, which achieved relatively high performance, began to misclassify new samples as the gradual growth of encapsulation tissue and progressive electrode movement were simulated. In contrast to this observed decrease, the performance of the baseline calibration approach at the lowest SNR of $-15 \mathrm{~dB}$ was relatively stable in both simulations, albeit at a lower level of performance. It is possible that the noisier training data may have led to a network that was better able to generalize and therefore suffered less of a decline as additional signal distortions occurred. Nonetheless, these observations supported the hypothesis that selective recording performance would decline over time, thus calling for strategies to compensate for these changes.

\subsection{Periodic Recalibration}

The periodic recalibration strategy led to a consistent pattern across all noise levels in the encapsulation tissue simulation. The classification performance initially increased at time point ET1 and decreased at time point ET2 before finally increasing once again at the final time point, ET3. This result may provide evidence to suggest that the recorded signals from different pathways may be more easily characterized and discriminated when the encapsulation tissue fully fills the space between the epineurium and the cuff electrode, as in time point ET3. Potential mechanisms for this trend could be the increased CAP amplitude observed in our simulated recordings when the space between the cuff and nerve was filled with encapsulation tissue and decreased shunting effects from the saline layer. Previously, Moffit et al. modeled a microelectrode array in the presence of encapsulation tissue growth [59] and reported an increase in signal amplitude that was directly proportional to the thickness of the modeled encapsulation tissue, a result that offered a possible explanation to observations of signal increases seen experimentally by Vetter et al [60]. While referring to a different neural interface, these results were consistent with our findings here and support the validity of our models.

In summary, both simulations provide evidence that a network periodically retrained with new and explicitly labelled samples can adapt effectively to changes in bioelectric signal characteristics during chronic implantation.

Practically, periodic recalibration would require action on the part of the end user, as well as the possible involvement of a clinician or other support personnel, and therefore reduce the convenience of the system. It is difficult to make an accurate estimation of the frequency of recalibration that would be needed for this technique to be effective based on the results of this study, especially considering the fact that other chronic studies mentioning nerve cuff electrode encapsulation tissue growth in vivo $[15,20,43]$ only assessed the degree of encapsulation tissue formation at the end of their studies (anywhere between 9 and 80 weeks post-implantation). That being said, however, there has been mention of abnormal tissue growth and fiber morphology [20], as well as the modification of the electrical properties associated with nerve cuff electrodes [61] seen as early as two weeks post-implantation. Therefore, it is not unlikely that a recalibration procedure may be required every two-to-four weeks in order to be effective, at least in the early stages. Considering this fact, employing a periodic recalibration approach would likely lessen the overall usability of a system that implements a neural interface with a selective recording algorithm, which motivates the use of a self-learning approach to minimize human intervention.

\subsection{Self-Learning Approach}

The degradation over time observed with the self-learning approach could be explained by the fact that the samples used to retrain the network in this approach were labelled by the network itself, and only those with high enough confidence were used to create a training dataset. For this reason, the training set used in this approach was often smaller than that of the other two updating approaches. Though the samples incorporated into the self-labelled datasets were based on confidence level, this did not necessarily 
prevent the network from including incorrectly labelled samples into the training sets used, and, as such, an accumulation of incorrectly labelled samples began to occur over time. This is the most likely explanation for the degradation trend seen in the self-learning approach and indicates that additional refinements to this updating approach are required in order to improve its efficacy. Like the baseline calibration approach, this trend was more obvious at higher signal-to-noise ratios of -5 and $-10 \mathrm{~dB}$, whereas the performance of the self-learning approach was relatively more stable at the lowest $\mathrm{SNR}$ of $-15 \mathrm{~dB}$.

A pattern observed in both the baseline calibration and self-learning approaches was that performance was relatively stable until a rotation of $25^{\circ}$, at which point the performance began to decline more sharply. This trend was consistent across all three investigated noise levels. It is possible that this phenomenon was due to the geometry of the cuff, as contacts were spaced or located at intervals of $45^{\circ}$. Thus, at a rotation of $20^{\circ}$, the current position of each contact was closer to its original location compared to the original position of another contact. Once rotated $25^{\circ}$ or greater, contacts were closer to the original location of some other contact. Because of this, the CNN may have misinterpreted the spatiotemporal signatures, possibly leading to the decline in performance.

In summary, in both scenarios, a network that achieved a relatively high performance upon initial training at baseline and then used a self-learning update strategy began to misclassify new samples as we simulated the gradual growth of encapsulation tissue and progressive electrode movement. We therefore conducted additional analysis of the factors that could influence self-learning performance.

\subsection{Influence of Training Frequency and Initial Performance on Self-Learning Approach Performance}

Two major factors thought to influence the efficacy of the self-learning approach were investigated in this simulation: the frequency of training and the initial performance level. In order to quantify the effects of these factors on this strategy, the self-learning approach was applied at six different initial starting performances across three noise levels. At each of these initial performance levels, each training frequency (one-time, two-times, four-times, and eight-times) was applied, as shown in Figure 10.

Figure 10 illustrates a few important points about the self-learning approach. When starting at the higher initial performance level, the difference between the self-learning approach and the control condition decreased along with the SNR. In contrast, for the lower initial performance level, the control condition always outperformed the self-learning approach. Furthermore, in the case of lower initial performance levels, more frequent training generally led to worse performance over time, thus supporting the hypothesis of an error accumulation phenomenon.

In the final step of this analysis, Figure 11 shows the relationship between the rate of decline, the initial performance level, the frequency of training, and the level of noise. These data highlight specific cases in which the self-learning approach was able to slow the performance decline over time. Specifically, in the case of recordings of relatively high SNRs in the context of nerve cuff recordings, implementing the self-learning approach at a high initial performance, with frequent retraining, could slow the decline of the selective recording algorithm's performance over time as a result of encapsulation tissue growth. The implications of this are that a system implementing a self-learning approach may require less frequent retraining, ultimately improving both its convenience and usability.

\section{Conclusions}

This simulation study confirms that the performance of a selective recording approach, based on multi-contact nerve cuff recordings and a CNN trained at baseline, will degrade over time, and it demonstrates strategies to compensate for this trend. A periodic recalibration approach could help maintain or even improve selective recording performance over time in the presence of chronic implantation challenges (encapsulation tissue and electrode movement) at the cost of increased demands on the user. A self-learning update approach in which an algorithm is retrained at specific points in time with new, self-labelled data, 
will, in most cases, fail over time as a result of an eventual accumulation of mislabeled training data. However, under specific conditions (a high SNR, high initial performance, and frequent retraining), this strategy may help to slow the rate of decline of selective recording performance compared to a control condition. These findings will assist in the development of more intuitive, robust, and longer-lasting closed-loop neuroprosthetic and neuromodulation applications. In particular, the practical implications of this research are to form the basis for establishing recalibration schedules and to implement automated self-learning techniques that reduce the necessary recalibration frequency.

Author Contributions: S.S. carried out the simulations, analysis, and drafted the manuscript. R.G.L.K. contributed to the development of the modeling and machine learning methods. S.S. and J.Z. conceived the study. All authors reviewed and approved the manuscript. All authors have read and agreed to the published version of the manuscript.

Funding: This work was supported in part by the Canadian Institutes of Health Research, the Toronto Rehabilitation Institute, and the Natural Sciences and Engineering Research Council of Canada (RGPIN-2014-05498, RGPIN-2020-06246).

Institutional Review Board Statement: Not applicable.

Informed Consent Statement: Not applicable.

Data Availability Statement: The data presented in this study are available on request from the corresponding author.

Conflicts of Interest: The authors declare no conflict of interest.

\section{References}

1. Dhillon, G.S.; Lawrence, S.M.; Hutchinson, D.T.; Horch, K.W. Residual function in peripheral nerve stumps of amputees: Implications for neural control of artificial limbs. J. Hand Surg. 2004, 29, 605-615. [CrossRef] [PubMed]

2. Dhillon, G.S.; Krüger, T.B.; Sandhu, J.S.; Horch, K.W. Effects of short-term training on sensory and motor function in severed nerves of long-term human amputees. J. Neurophysiol. 2005, 93, 2625-2633. [CrossRef] [PubMed]

3. Davis, T.S.; Wark, H.A.C.; Hutchinson, D.T.; Warren, D.J.; O’Neill, K.; Scheinblum, T.; Clark, G.A.; Normann, R.A.; Greger, B. Restoring motor control and sensory feedback in people with upper extremity amputations using arrays of 96 microelectrodes implanted in the median and ulnar nerves. J. Neural Eng. 2016, 13. [CrossRef]

4. Micera, S.; Citi, L.; Rigosa, J.; Carpaneto, J.; Raspopovic, S.; di Pino, G.; Rossini, L.; Yoshida, K.; Denaro, L.; Dario, P.; et al. Decoding information from neural signals recorded using intraneural electrodes: Toward the development of a neurocontrolled hand prosthesis. Proc. IEEE 2010, 98, 407-417. [CrossRef]

5. Inmann, A.; Haugland, M. Functional evaluation of natural sensory feedback incorporated in a hand grasp neuroprosthesis. Med. Eng. Phys. 2004, 26, 439-447. [CrossRef] [PubMed]

6. Haugland, M.K.; Sinkjær, T. Cutaneous whole nerve recordings used for correction of footdrop in hemiplegic man. IEEE Trans. Rehabil. Eng. 1995, 3, 307-317. [CrossRef]

7. Plachta, D.T.T.; Gierthmuehlen, M.; Cota, O.; Espinosa, N.; Boeser, F.; Herrera, T.C.; Stieglitz, T.; Zentner, J. Blood pressure control with selective vagal nerve stimulation and minimal side effects. J. Neural Eng. 2014, 11, 036011. [CrossRef] [PubMed]

8. Harreby, K.R.; Sevcencu, C.; Struijk, J.J. Early seizure detection in rats based on vagus nerve activity. Med. Biol. Eng. Comput. 2011, 49, 143-151. [CrossRef]

9. Nielsen, T.N.; Struijk, J.J.; Harreby, K.R.; Sevcencu, C. Early detection of epileptic seizures in pigs based on vagus nerve activity. In Converging Clinical and Engineering Research on Neurorehabilitation; Springer: Berlin, Germany, 2013.

10. Cloutier, A.; Yang, J. Design, control, and sensory feedback of externally powered hand prostheses: A literature review. Crit. Rev. Biomed. Eng. 2013, 41. [CrossRef]

11. Del Valle, J.; Navarro, X. Interfaces with the peripheral nerve for the control of neuroprostheses. Int. Rev. Neurobiol. 2013, 109, 63-83.

12. Brindley, G.S. The first 500 patients with sacral anterior root stimulator implants: General description. Paraplegia 1994, 32, 795-805. [CrossRef] [PubMed]

13. Lyons, G.M.; Sinkjær, T.; Burridge, J.H.; Wilcox, D.J. A review of portable FES-based neural orthoses for the correction of drop foot. IEEE Trans. Neural Syst. Rehabil. Eng. 2002, 10, 260-279. [CrossRef] [PubMed]

14. Waters, R.L.; McNeal, D.R.; Faloon, W.; Clifford, B. Functional electrical stimulation of the peroneal nerve for hemiplegia. Long-term clinical follow-up. J. Bone Jt. Surg. Ser. A 1985, 67, 792-793. [CrossRef]

15. Dweiri, Y.M.; Eggers, T.E.; Gonzalez-Reyes, L.E.; Drain, J.; McCallum, G.A.; Durand, D.M. Stable detection of movement intent from peripheral nerves: Chronic study in dogs. Proc. IEEE 2017, 105, 50-65. [CrossRef] 
16. Stein, R.B.; Nichols, T.R.; Jhamandas, J.; Davis, L.; Charles, D. Stable long-term recordings from cat peripheral nerves. Brain Res. 1977, 128, 21-38. [CrossRef]

17. Popović, D.B.; Stein, R.B.; Jovanović, K.L.; Dai, R.; Kostov, A.; Armstrong, W.W. Sensory nerve recording for closed-loop control to restore motor functions. IEEE Trans. Biomed. Eng. 1993, 40, 1024-1031. [CrossRef] [PubMed]

18. Struijk, J.J.; Thomsen, M.; Larsen, J.O.; Sinkjaer, T. Cuff electrodes for long-term recording of natural sensory information. IEEE Eng. Med. Biol. Mag. 1999, 18, 91-98. [CrossRef]

19. Rodriguez, F.J; Ceballos, D.; Schu, M.; Valero, A.; Valderrama, E.; Stieglitz, T.; Navarro, X. Polyimide cuff electrodes for peripheral nerve stimulation. J. Neurosci. Methods 2000, 98, 105-118. [CrossRef]

20. Grill, W.M.; Mortimer, J.T. Neural and connective tissue response to long-term implantation of multiple contact nerve cuff electrodes. J. Biomed. Mater. Res. 2000, 50, 215-226. [CrossRef]

21. Vasudevan, S.; Patel, K.; Welle, C. Rodent model for assessing the long term safety and performance of peripheral nerve recording electrodes. J. Neural Eng. 2017, 14, 16008. [CrossRef]

22. Christie, B.P.; Freeberg, M.; Memberg, W.D.; Pinault, G.J.; Hoyen, H.A.; Tyler, D.J.; Triolo, R.J. Long-term stability of stimulating spiral nerve cuff electrodes on human peripheral nerves. J. Neuroeng. Rehabil. 2017, 14, 1-12. [CrossRef] [PubMed]

23. Navarro, X.; Valderrama, E.; Stieglitz, T.; Schüttler, M. Selective fascicular stimulation of the rat sciatic nerve with multipolar polyimide cuff electrodes. Restor. Neurol. Neurosci. 2001, 18, 9-21. [PubMed]

24. Tarler, M.D.; Mortimer, J.T. Selective and independent activation of four motor fascicles using a four contact nerve-cuff electrode. IEEE Trans. Neural Syst. Rehabil. Eng. 2004, 12, 251-257. [CrossRef] [PubMed]

25. Veraart, C.; Grill, W.M.; Mortimer, J.T. Selective control of muscle activation with a multipolar nerve cuff electrode. IEEE Trans. Biomed. Eng. 1993, 40, 640-653. [CrossRef] [PubMed]

26. Walter, J.S.; Griffith, P.; Sweeney, J.; Scarpine, V.; Bidnar, M.; McLane, J.; Robinson, C. Multielectrode nerve cuff stimulation of the median nerve produces selective movements in a raccoon animal model. J. Spinal Cord Med. 1997, 20, 233-243. [CrossRef] [PubMed]

27. Grill, W.M.; Mortimer, J.T. The effect of stimulus pulse duration on selectivity of neural stimulation. IEEE Trans. Biomed. Eng. 1996, 43, 161-166. [CrossRef]

28. Raspopovic, S.; Carpaneto, J.; Udina, E.; Navarro, X.; Micera, S. On the identification of sensory information from mixed nerves by using single-channel cuff electrodes. J. Neuroeng. Rehabil. 2010, 7, 17. [CrossRef]

29. Tesfayesus, W.; Durand, D.M. Blind source separation of peripheral nerve recordings. J. Neural Eng. 2007, 4, S157. [CrossRef]

30. Metcalfe, B.; Chew, D.; Clarke, C.; Donaldson, N.; Taylor, J. Fibre-selective discrimination of physiological ENG using velocity selective recording: Report on pilot rat experiments. In Proceedings of the 2014 36th Annual International Conference of the IEEE Engineering in Medicine and Biology Society EMBC 2014, Chicago, IL, USA, 26-30 August 2014; pp. $2645-2648$.

31. Metcalfe, B.W.; Chew, D.J.; Clarke, C.T.; Donaldson, N.D.N.; Taylor, J.T. A new method for spike extraction using velocity selective recording demonstrated with physiological ENG in Rat. J. Neurosci. Methods 2015, 251, 47-55. [CrossRef]

32. Metcalfe, B.; Nielsen, T.; Taylor, J. Velocity selective recording: A demonstration of effectiveness on the vagus nerve in pig. In Proceedings of the Annual International Conference of the IEEE Engineering in Medicine and Biology Society EMBS, Honolulu, HI, USA, 18-21 July 2018; Volume 2018, pp. 2945-2948.

33. Schuettler, M.; Donaldson, N.; Seetohul, V.; Taylor, J. Fibre-selective recording from the peripheral nerves of frogs using a multi-electrode cuff. J. Neural Eng. 2013, 10, 036016. [CrossRef]

34. Yoshida, K.; Kurstjens, G.A.M.; Hennings, K. Experimental validation of the nerve conduction velocity selective recording technique using a multi-contact cuff electrode. Med. Eng. Phys. 2009, 31, 1261-1270. [PubMed]

35. Wodlinger, B.; Durand, D.M. Recovery of neural activity from nerve cuff electrodes. In Proceedings of the 2011 Annual International Conference of the IEEE Engineering in Medicine and Biology Society, Boston, MA, USA, 30 August-3 September 2011; pp. 4653-4656.

36. Eggers, T.E.; Dweiri, Y.M.; McCallum, G.A.; Durand, D.M. Model-based Bayesian signal extraction algorithm for peripheral nerves. J. Neural Eng. 2017, 14, 056009. [PubMed]

37. Wodlinger, B.; Durand, D.M. Localization and recovery of peripheral neural sources with beamforming algorithms. IEEE Trans. Neural Syst. Rehabil. Eng. 2009, 17, 461-468. [PubMed]

38. Zariffa, J.; Popovic, M.R. Localization of active pathways in peripheral nerves: A simulation study. IEEE Trans. Neural Syst. Rehabil. Eng. 2009, 17, 53-62. [PubMed]

39. Zariffa, J.; Nagai, M.K.; Schuettler, M.; Stieglitz, T.; Daskalakis, Z.J.; Popovic, M.R. Use of an experimentally derived leadfield in the peripheral nerve pathway discrimination problem. IEEE Trans. Neural Syst. Rehabil. Eng. 2011, 19, 147-156. [PubMed]

40. Koh, R.G.L.; Nachman, A.I.; Zariffa, J. Use of spatiotemporal templates for pathway discrimination in peripheral nerve recordings: A simulation study. J. Neural Eng. 2017, 14, 016013. [PubMed]

41. Koh, R.G.L.; Nachman, A.I.; Zariffa, J. Classification of naturally evoked compound action potentials in peripheral nerve spatiotemporal recordings. Sci. Rep. 2019, 9, 11145.

42. Koh, R.G.L.; Balas, M.; Nachman, A.I.; Zariffa, J. Selective peripheral nerve recordings from nerve cuff electrodes using convolutional neural networks. J. Neural Eng. 2020, 17, 016042.

43. Grill, W.M.; Mortimer, J.T. Electrical properties of implant encapsulation tissue. Ann. Biomed. Eng. 1994, $22,23-33$. 
44. Polikov, V.S.; Tresco, P.A.; Reichert, W.M. Response of brain tissue to chronically implanted neural electrodes. J. Neurosci. Methods 2005, 148, 1-18.

45. Merrill, D.R.; Tresco, P.A. Impedance characterization of microarray recording electrodes in vitro. IEEE Trans. Biomed. Eng. 2005, 52, 1960-1965. [PubMed]

46. Garai, P.; Koh, R.G.L.; Schuettler, M.; Stieglitz, T.; Zariffa, J. Influence of anatomical detail and tissue conductivity variations in simulations of multi-contact nerve cuff recordings. IEEE Trans. Neural Syst. Rehabil. Eng. 2017, 25, 1653-1662. [CrossRef] [PubMed]

47. Silveira, C.; Brunton, E.; Spendiff, S.; Nazarpour, K. Influence of nerve cuff channel count and implantation site on the separability of afferent ENG. J. Neural Eng. 2018, 15, 046004. [CrossRef]

48. CIBC. Seg3D: Volumetric Image Segmentation and Visualization; Scientific Computing and Imaging Institute (SCI): Salt Lake City, UT, USA, 2016.

49. Fang, Q.; Boas, D.A. Tetrahedral mesh generation from volumetric binary and grayscale images. In Proceedings of the 2009 IEEE International Symposium on Biomedical Imaging: From Nano to Macro, ISBI, Boston, MA, USA, 28 June-1 July 2009.

50. Scientific Computing and Imaging Institute (SCI). SCIRun: A Scientific Computing Problem Solving Environment; Scientific Computing and Imaging Institute (SCI): Salt Lake City, UT, USA, 2015.

51. Weinstein, D.; Zhukov, L.; Johnson, C. Lead-field bases for electroencephalography source imaging. Ann. Biomed. Eng. 2000, 28, 1059-1065. [CrossRef] [PubMed]

52. Yoo, P.B.; Durand, D.M. Selective recording of the canine hypoglossal nerve using a multicontact flat interface nerve electrode. IEEE Trans. Biomed. Eng. 2005, 52, 1461-1469. [CrossRef] [PubMed]

53. Parrini, S.; Delbeke, J.; Romero, E.; Legat, V.; Veraart, C. Hybrid finite elements and spectral method for computation of the electric potential generated by a nerve cuff electrode. Med. Biol. Eng. Comput. 1999, 37, 733-736. [CrossRef]

54. Schwarz, J.R.; Reid, G.; Bostock, H. Action potentials and membrane currents in the human node of Ranvier. Pflügers Arch. Eur. J. Physiol. 1995, 430, 283-292. [CrossRef]

55. Struijk, J.J.; Thomsen, M. Tripolar nerve cuff recording: Stimulus artifact, EMG and the recorded nerve signal. In Proceedings of the 17th International Conference of the Engineering in Medicine and Biology Society, Montreal, QC, Canada, 20-23 September 1995; Volume 2, pp. 1105-1106.

56. Abadi, M.; Barham, P.; Chen, J.; Chen, Z.; Davis, A.; Dean, J.; Devin, M.; Ghemawat, S.; Irving, G.; Isard, M.; et al. TensorFlow: A system for large-scale machine learning. In Proceedings of the 12th USENIX Conference on Operating Systems Design and Implementation, Savannah, GA, USA, 2-4 November 2016; Volume 16, pp. 265-283.

57. Schwemmer, M.A.; Skomrock, N.D.; Sederberg, P.B.; Ting, J.E.; Sharma, G.; Bockbrader, M.A.; Friedenberg, D.A. Meeting brain-computer interface user performance expectations using a deep neural network decoding framework. Nat. Med. 2018, 24, 1669-1676. [CrossRef]

58. Zariffa, J.; Nagai, M.K.; Daskalakis, Z.J.; Popovic, M.R. Influence of the number and location of recording contacts on the selectivity of a nerve cuff electrode. IEEE Trans. Neural Syst. Rehabil. Eng. 2009, 17, 420-427.

59. Moffitt, M.A.; McIntyre, C.C. Model-based analysis of cortical recording with silicon microelectrodes. Clin. Neurophysiol. 2005, 116, 2240-2250. [CrossRef]

60. Vetter, R.J.; Williams, J.C.; Hetke, J.F.; Nunamaker, E.A.; Kipke, D.R. Chronic neural recording using silicon-substrate microelectrode arrays implanted in cerebral cortex. IEEE Trans. Biomed. Eng. 2004, 51, 896-904. [CrossRef] [PubMed]

61. Grill, W.M.; Mortimer, J.T. Temporal stability of nerve cuff electrode recruitment properties. In Proceedings of the Annual International Conference of the IEEE Engineering in Medicine and Biology Society (EMBC), Montreal, QC, Canada, 20-23 September 1995; Volume 17, pp. 1089-1090. 